\title{
Experimental Ferrogravitational Field around Untwisting Closed Superconductor
}

\author{
Robert A. Sizov \\ Individual Researcher, Moscow, Russia \\ Email: sizov.robert@gmail.com
}

How to cite this paper: Sizov, R.A. (2020) Experimental Ferrogravitational Field around Untwisting Closed Superconductor. Journal of Modern Physics, 11, 1807-1826. https://doi.org/10.4236/jmp.2020.1111113

Received: October 15, 2020

Accepted: November 10, 2020

Published: November 13, 2020

Copyright (C 2020 by author(s) and Scientific Research Publishing Inc. This work is licensed under the Creative Commons Attribution International License (CC BY 4.0).

http://creativecommons.org/licenses/by/4.0/

\begin{abstract}
The study by the author of magnetic scattering neutrons in the structures of ferrimagnets, as well as his experiments with the separation of magnetic charges in dipole pairs $\pm \mathrm{g}$ in magnetic field, showed that fundamental magnetic particles (magnetic charges) are real structural components of atoms and substance. It is the magnetic poles, and not the moving electric charges are the direct sources of all magnetic fields and magnetic manifestations in Nature. Basic reasons of ignoring the magnetic fundamental particles by world physical theory, for almost 150 years, are the ultra-harsh confinement of these particles in substance which radically is different from the confinement electrons, as well as the vicious concept of the electric magnetism Maxwell. Rotating magnetic dipoles in conductors which are untwisted by electric current, are direct sources of the vortex magnetic field $\operatorname{rot} H$. One should also expect the formation of a vortex electric field $\operatorname{rot} E$ forming by rotating electric dipoles which are untwisted by the current of magnetic charges. This article provides an experimental answer to the question: what field is formed around a conductor if joint direct currents of electric $J_{\mathrm{e}}$ and magnetic $J_{\mathrm{g}}$ charges are passed through it? The author's experiments have shown that in this case the vortex electromagnetic current is realized which manifests itself as the vortex electromagnetic (gravitational) field. It is possible to implement such a process, according to the results of the author's research, exclusively in superconductors. The vector character of the gravitational field is in many respects similar to the vortex magnetic field which makes it possible to introduce such it states as paragravitation and ferrogravitation into representations. To create joint currents of electric and magnetic charges, the author used the inertial forces of these particles under conditions of acceleration and deceleration of the rotational motion of the closed lead superconductor. The result of this experiment was the gravitational, as it turned out later, the ferrogravitational field, which was detected by effect repulsion of trial cargos from the coil with a superconducting winding at the stage of its untwist. The
\end{abstract}


latter process is defined by the author as an effect of the gravitational (ferrogravitational) levitation. The values of ferrogravitational (levitational) forces noted in this experiment were: $120 \mathrm{mg}$ for a tungsten trial cargo and 50 and $25 \mathrm{mg}$ for a lead cargo with an error of $\pm 15 \mathrm{mg}$. The values of ferrogravitational (levitational) forces noted in this experiment are: $120 \mathrm{mg}$ for from tungsten trial cargo and 50 and $25 \mathrm{mg}$ for a cargo from lead, with an error of $\pm 15 \mathrm{mg}$. The "anomaly" noted by the author in this study was in the absence of any absence of a gravitational effect on stage a braking of the coil. Probable cause of the noted "anomaly" is discussed in the Discussion of Results chapter.

\section{Keywords}

Magnetic Charges, Magnetons, Antimagnetons, Magnetic Dipoles, True

Antielectrons, S-Gravitons, Gravitational Field, Ferro- and Paragravitation, Gravitational “Dark Energy”, Gravitational Levitation

\section{Introduction to the Physics of Magnetic Charges and True Antielectrons in Composition of Substance}

The author's experimental and theoretical studies which have been carried out since 1968 have shown that magnetic poles (magnetic charges) are real structural components of atoms and substance [1] [2] [3] [4] [5]. It is magnetic charges, but not moving electrons are the direct sources of all magnetic fields and magnetic manifestations in Nature. The charges of magnetic spinor particles (g) which together with electrons and true antielectrons form a shells of atoms, correspond condition $\mathrm{g}=\mathrm{e}$, where $\mathrm{e}$ is the electron charge. Magnetic charge with negative sign g-received the author's name magneton (not to be confused with the so-called magneton of Bohr). By antiparticle to a magneton is the antimagneton with positive magnetic charge $\mathrm{g}^{+}$. The main state of fundamental magnetic charges in Nature, as well as electric charges, is its existence in composition of the corresponding bispinors, which most often appear in the form of dipoles [6].

Note 1. The bispinor is a pair of spinor particles, i.e. charged particles, in the composition: a spinor and a corresponding antispinor. Spinors are, for example, electrons and magnetons. Antispinors are fundamental particles with a positive charge, such as true antielectrons and antimagnetons. In the absence of external influences the bispinor, as a result of close contact in a pair of constituent it particles, is in a state complete closure a spins. In this case, complete mutual compensation of the spinor fields of the particles that make up the bispinor is realized, which, in this state, does not manifest itself by the external spinor fields, i.e. are practically undetectable. It is the noted effect of complete spin closure in the interaction of an electron with a positron that is the cause of such a global theoretical delusion as the annihilation of particles in a particle-antiparticle pair.

Under the influence of the electric current $J_{\mathrm{e}}$ the vortices of the above-mentioned magnetic dipoles are realized in the conductor which is described by the expres- 
sion $\operatorname{rot} J_{\mathrm{g}}$, and the equation of the process of their formation has the form:

$$
k_{1} \boldsymbol{J}_{\mathrm{e}}=\operatorname{rot} \boldsymbol{J}_{\mathrm{g}} .
$$

It is the vortices of magnetic charges formed in conductor under the influence of an electric current are direct sources of the vortex magnetic field $\operatorname{rot} H$, the formation of which is described by the equation

$$
k_{2} \operatorname{rot} \boldsymbol{J}_{\mathrm{g}}=\operatorname{rot} \boldsymbol{H} .
$$

The well-known, so-called first Maxwell's equation

$$
k \boldsymbol{J}_{\mathrm{e}}=\operatorname{rot} \boldsymbol{H},
$$

is extremely erroneous, since it combines into a single action two physically different processes mentioned above. This equation appeared as a result of the superficial impression of the Great Theoretician from the famous Experience of Oersted [7].

Unfortunately, at the time of the formation of his vicious electromagnetic concept and the introduction of the super-harmful Equation (3) in 1873, Maxwell, apparently, did not even assume that real magnetic poles could exist in Nature. It is also important to emphasize here that moving electric charges in the described process (1) are just intermediaries, whose role is, exclusively, in the unwinding of vortices of magnetic dipoles actually existing in the conductor [8] [9]. The aforementioned Great misconception by Maxwell led to numerous errors in physical science, giving rise, for example, to such a vicious theoretical direction as relativism.

The first person to experimentally observe real magnetic charges in a substance was the remarkable Austrian physicist Felix Ehrenhaft. Based on the results of his research which lasted for more than 40 years, he published about 30 articles in physical journals [10]. It is important to note that Ehrenhaft's experiments were repeated by numerous followers who confirmed his results and conclusions (see, for example [11]).

The Ehrenhaft experiments are a magnetic analog of well-known Milliken tests from determining the value of the electron charge. Very small particles of solids substance were placed in a vertical uniform magnetic field free of residual electrical charges. The particles were illuminated by concentrated beam of light. The optical system allowed to determine the parameters of the particles movement. The basic experimental result of investigation Ehrenhaft consisted in the fact that it was found a logical movement of particles along power lines of the magnetic field. With the change in the direction of the field, the direction of movement of the particles also was changed. According to conclusions Ehrenhaft motions of particles, that observed in his experiments, are determined their charging by the magnetic charges of different signs.

Experimental and theoretical studies of the author, with compelled interruptions, have been carried out since 1968 have shown that electrons and magnetic charges in a normal conductor are under conditions of radically different phys- 
ics of their confinement. Electrons populate real conduction bands which allow them to participate in the composition of the electric current. As for magnetic charges that are not included in atomic structures, they are in potential zones of conduction and their movement in a normal conductor is possible only in the processes of the noted rotations around the lines of electric current.

However, the situation with the confinement of magnetic charges in conductors, changes dramatically under conditions of superconductivity when these charges leave potential zones and pass into real conduction zones. In this case, magnetic dipoles, in the process of their rotation around the electric current lines, do not experience "friction" against the superconductor lattice, what and determines its zero electrical resistance or superconducting effect. It is important to note that true anti-electrons $\left(\mathrm{e}^{+}\right)$, along with the magnetic charges, as well as are in substance under hard confinement conditions.

The author's research has shown that the positron is not an anti-electron, as it follows from modern physical concepts, since represents the mass, i.e. atomic-like structure. As for the true antielectron, it "sits" in the positron as a nucleus and determines its positive charge $\mathrm{e}^{+}$[12] (see also [6]).

The conditions for the confinement of electric and magnetic charges in a substance are presented in Figure 1, which shows the layout of the levels of electrons, true anti-electrons and magnetic charges (magnetons and anti-magnets) on the energy scale. As you know, electronic levels are in a potential "well" and for an electron to come out into a free state from connection with the conductor lattice, it is necessary to increase its internal energy, for example, by heating. As for the magnetic charges and true anti-electrons, their energy levels are located under the potential "dome" and for these particles to enter the free state, the substance should be cooled, i.e. reduce his internal energy.

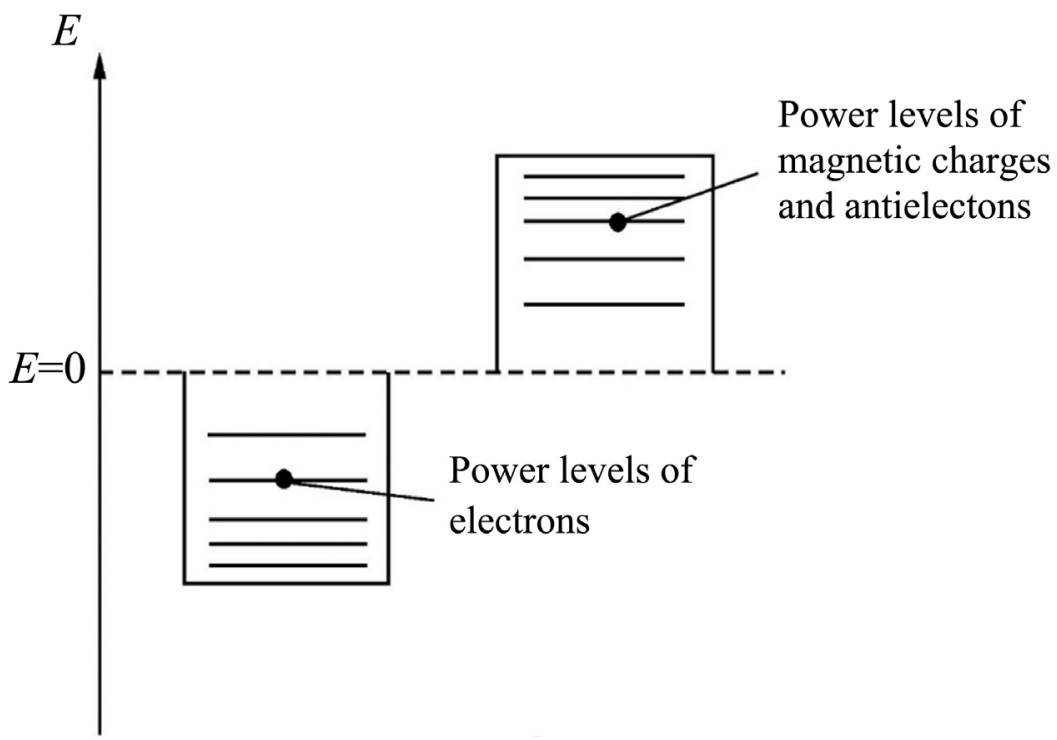

Figure 1. Schematic diagram of the location on the energy scale of the levels of electrons (in the energy well) and magnetic charges with true anti-electrons (under the energy dome). 
At deep stages of cooling of some substances, their superconducting state is realized, which associated with exit of magnetic charges and true antielectrons into a state free from coupling with the superconductor lattice. It is the special conditions of the confinement of magnetic spinor particles, as well as of true antielectrons in substance noted above that played a very "cruel joke" with the physical theory, which manifested itself, for example, in the form vicious electric magnetism of Maxwell.

It is important to note that the vicious ignoring of real magnetic charges, as well as true electrons, did not pass without a trace for the existing physical theory. These unrecognized fundamental particles were perforce replaced, more or less, by suitable theoretical surrogates. For example, magnetic charges in atomic shells were substitute with such "fig leaves" as the magnetic moments of electrons ( $3 \mathrm{~d}$ or $4 \mathrm{f}$ ), and the true antielectrons by Dirac holes or electron vacancies [13] [14].

According to the author of the article, the famous Dirac monopole should also be referred to the above-mentioned surrogate-substitutes for real elementary particles. The position taken by Dirac in questions related to magnetic poles is more than strange. On the one hand, he argues that it would be surprising if nature did not use this possibility, that is, the realization of magnetic poles in the structures of substance and showed, that quantum electromagnetic theory fully admits their existence. But, on the other hand, it fills its monopole with a magnetic field, which is created in ultra-thin filaments-solenoids fed by electric current. Thus, if to remove the filaments-solenoids of Dirac, i.e. if you disconnect the monopole from the source of electric current, then the magnetic monopole will not be able to be realized. In other words, despite lofty goals, Dirac and his magnetic monopoly failed to avoid being infected by the vicious Maxwell virus. In addition, the magnetic field emitted by the Dirac monopole can only be a vortex field, which is described by vortex vector $\operatorname{rot} H$, since only such a magnetic field can be formed around a conductor with a constant electric current. It is also important to note that Dirac did not associate his monopole with magnetic fields and magnetic manifestations which surround man, because he did not see any prospects for this [15].

At the end of the seventies (1979), the author asked himself an interesting question, what can happen if we pass joint linear currents of electric and magnetic charges through a superconductor? Of course, there are no problems with the implementation of electric current, but where to get the source of the current of magnetic charges? The solution to this problem was the use of inertial forces of magnetic and electric charges in the conditions of untwist or braking a coil with a closed winding from of the superconducting wire. The author assumed that if you spin a closed loop of a superconductor and then abruptly stop its rotation, then due to inertial forces in the superconductor, joint currents of electric and magnetic charges are realized, i.e. an electromagnetic current is generated. In this process, as the author assumed, these currents will not remain linear, but their mutual twisting will occur with the formation of an eddy electromagnetic 
current, which, most likely, will manifest itself as a vortex electromagnetic (gravitational) field.

The fact that magnetic charges have the property of inertia is evidenced, for example, by the effect of self-induction. The fact that after removing the electrical voltage in the conductor, for some time, the electric current is maintained, according to the author, is determined by the inertia of magnetic charges in the composition eddy dipole currents involved in the process of self-induction according to the equation $\operatorname{krot}_{\mathrm{g}}=J_{\mathrm{e}}[16]$.

\section{Experimental Ferrogravitational Field around Untwisting Closed Superconductor}

\subsection{Experimental Technique}

The schematic diagram of the setup on which dynamic experiments were performed to create joint currents of electric and magnetic charges in superconductors using inertial forces, is shown in Figure 2. The setup was a large helium cryostat (1), $140 \mathrm{~cm}$ high with reinforced wall of the inner (helium) vessel. In the lower part of the inner vessel, on a rolling bearing 2 , a coil 3 with windings consisted of lead wire 0.8 and $1 \mathrm{~mm}$ in diameter. As part of the coil windings, both one section with a wire with a diameter of $0.8 \mathrm{~mm}$ (Experiment I) and two

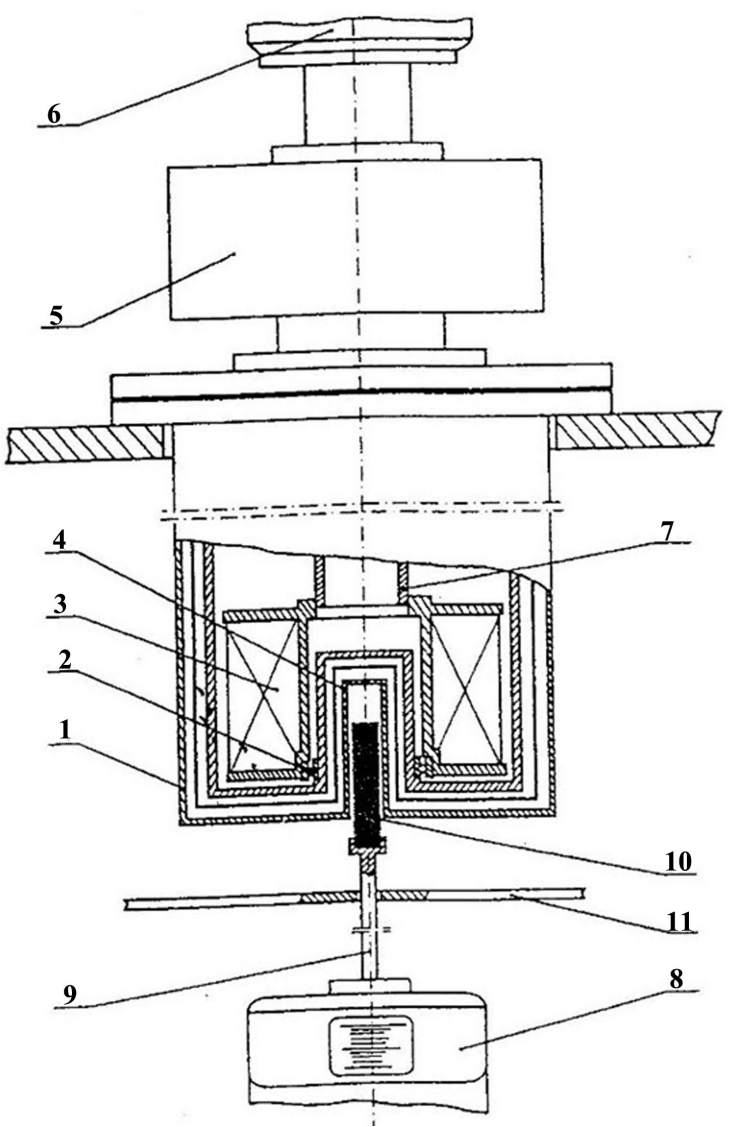

Figure 2. Schematic of experimental setup for obtaining a vortex electromagnetic (gravitational) field by means of a rotating superconductor. 
independent sections with a wire diameter of $1 \mathrm{~mm}$ (Experiment II) were implemented.

In order to connect the ends of the winding in each section, they were welded in an inert atmosphere. No surface insulation of the wire was made. The coil, after winding and securing the wire, was carefully balanced. The "appendix" 4 was made in the lower part of the cryostat, allowing the introduction of a "warm atmosphere" into the coil cavity. A wedge-type braking device 5 and an electric motor $6(1.5 \mathrm{~kW}$ and $2900 \mathrm{rpm})$ were installed on the cryostat cover. The transmission of rotation from the electric motor to the coil was carried out by means of a stainless steel pipe 7 .

The electromechanical balance 8 with a weighing accuracy of $\pm 10 \mathrm{mg}$ served as an indicator of the expected effect. On the movable part of the balance, on an aluminum rod 9, cylindrical weights 10 of tungsten and lead with a diameter of $16 \mathrm{~mm}$ and a length of $70 \mathrm{~mm}$ were fixed. The cargos were inserted into the "appendix" so that a third of their length would enter the coil cavity. To exclude parasitic influences on the balance mechanism, a screen 11 made of "Armco" iron sheets was installed between the cryostat and the balance.

The technology of the experiments was as follows.

After filling the cryostat with liquid helium to a level exceeding the top of the coil, the cryostat-coil system was held for $\sim 15$ minutes to equalize the temperature. Then the coil was spun up to the maximum engine speed. After holding in this mode for 10 - 15 seconds, the coil rotation was abruptly stopped by means of the braking device. The untwist time of the coil to the maximum speed was, according to the author's estimate, $2-2.5$ seconds, and the time the stop of rotation was $\sim 1.5$ seconds. The effect of changing the weight of the cylindrical cargos in the course of the experiment was recorded visually on a scale of the balance.

In total, two complete experiments were carried out, in which from 4 to 5 cycles were implemented (unwinding-braking). The limited number of experiments was explained, in particular, by the high consumption of liquid helium, especially in the dynamic part of the experiments. After each cycle, it was necessary to add helium again, cool and hold the system to equalize the temperature.

\subsection{Experimental Results}

Experiment I. The coil winding consisted of one closed section with lead wire of $0.8 \mathrm{~mm}$ in diameter. The total weight of lead in section is $14.2 \mathrm{~kg}$. In the course of the experiment, 4 cycles were performed (untwist-braking). In none of the noted cycles was there any change in the weight of the tungsten cargo, both at the stage of untwisting and at the stage of braking.

Experience II. The coil winding consisted of 2 independent sections with wire of diameter of $1 \mathrm{~mm}$. The total weight of the wire in the 2 coil sections was 15.3 kg. During the experiment 5 cycles were performed (untwisting-braking): 2 cycles with tungsten cargo and 3 cycles with lead cargo.

In none of the five cycles of the experiment was any reaction of the scales observed at the stage of braking the rotation of the coil, although it was at this stage 
that the appearance of the gravitational effect was expected. At the same time, at the stage of untwist the coil, a clear and very significant reaction of the balances was found. Two reliable changes in the weight of the cargos were noted: one in the 1st cycle of the experiment with a tungsten cargo $(\Delta \mathrm{P}=120 \pm 15 \mathrm{mg})$ and one in the $3 \mathrm{rd}$ cycle with a lead cargo $(\Delta \mathrm{P}=50 \pm 15 \mathrm{mg})$. The maximum error in estimating the change in the weight of the cargos $( \pm 15 \mathrm{mg})$ was adopted by us proceeding from the instrument error of the balance $( \pm 10 \mathrm{mg})$, plus a possible error $( \pm 5 \mathrm{mg})$ in the visual reading of $\Delta \mathrm{P}$ on the scale of the balance under the conditions of the fast dynamics of the experiment.

The noted effects, which, no doubt, were of a gravitational nature, were, at first, qualified by the author as the attraction of the weights to the coil, in accordance with the well-known law of universal gravitation. Problems with the sign of the observed gravitational effects, i.e. the attraction or repulsion of cargos that occurred in this pilot study are described in detail in the Discussion of Results below.

Changes in weights of the cargos from $\mathrm{W}$ and $\mathrm{Pb}$ which were obtained by the author in conditions of primary fixation of effect on visual observation of scale of the weights, are shown in Figure 3 by the dotted lines. In addition, in Figure 3 , by means of bold solid lines, the nature of these changes is shown after clarifying the sign of interactions between the cargos and coil using the results of subsequent experiments by the author. These latest results indicate that under the conditions of the described Experiment II only of the ferrogravitational field is realized around the superconductor. The essence of vector States of the gravitational

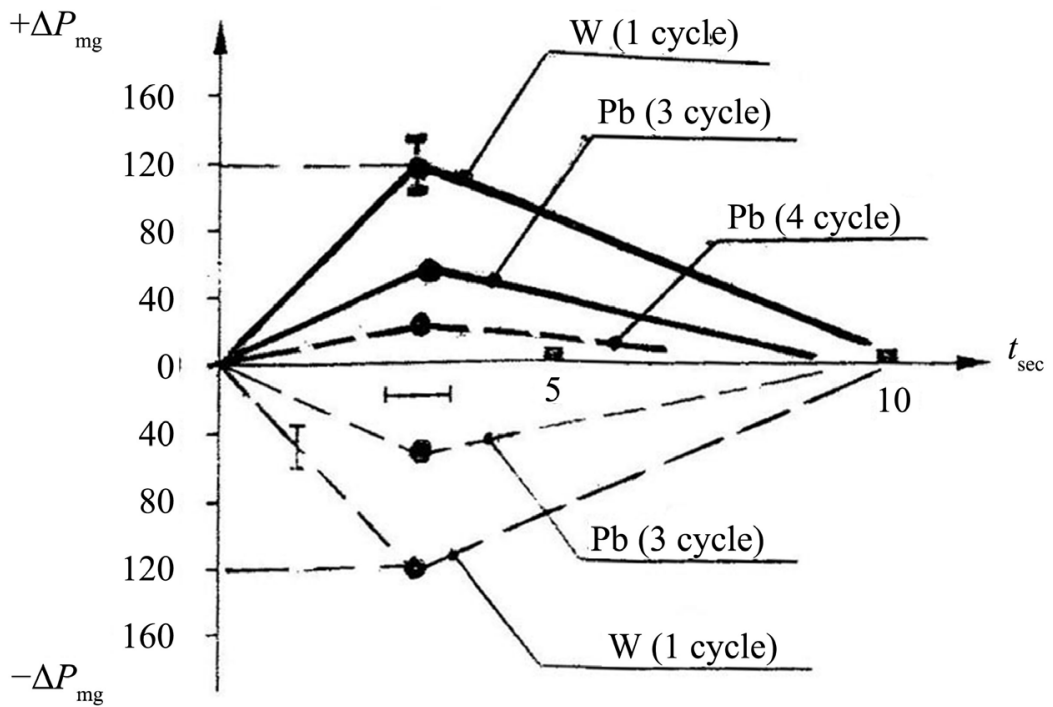

Figure 3. Values of changes in the weights $(\Delta \mathrm{P})$ of tungsten and lead cargos found in the author's experiments with rotating superconductors. The shows the nature and magnitude of the change in $\Delta \mathrm{P}$ of the weights (vertical axis) as a function of time $t$ from the moment the untwist began (plotted along the horizontal axis). A possible error in determining the time in the noted process is estimated by us as $\pm 0.5 \mathrm{sec}$. The values of reliable changes in weights of the cargos turned out to be very significant (120 and $50 \mathrm{mg}$ with an error of $\pm 15 \mathrm{mg}$ ). 
field formed based on the results of the author's experimental research, is presented in Section 4 of the article.

The behavior of the scales in the 4th cycle of the experiment with a lead weight turned out to be peculiar. In this cycle, it was not a decrease in the weight of the weights that was observed, but its increase, which corresponds to pushing the weight out of the coil cavity at the stage of untwist. The noted value of $\Delta \mathrm{P}$ in this case was $25 \pm 15 \mathrm{mg}$. The effect of the weight repulsion from the rotating coil was reliably detected as a result of the fact that the zero division of the scale of the balance fell into a single field of view together with the value $\Delta \mathrm{P}$ of the weight change.

In connection with the small value of this last effect and the strange sign of interaction, from the point of view of existing gravitational concepts, the author initially considered it as a kind of parasitic effect or "strange tendency". However, it was noticed that as the coil continued to spin, this "strange tendency" disappeared in about the same $\sim 10$ seconds as with the other weight changes noted above.

It is important to note, paradoxical as it may seem, but it was the last "tendency" that served as the basis for the author to revise his initial impressions of the described experiment and to conduct further experimental studies to clarify a real sign of the gravitational impact which was noted in the article.

In cycles 2 and 5 of Experiment II, no weight reactions were observed even at the trend level.

Note 2. The experiments with rotating superconductors presented in the article were carried out by the author in 1979-1981 on the territory of the Institute of Theoretical and Experimental Physics (building 7) in Moscow. The subsequent experiments of the author on the creation of a magneto-moving force (MMF) and constant current of magnetic charges $\left(J_{\mathrm{g}}\right)$ in superconductor, on the basis of which the s were clarified of the real signs of above the gravitational effects, were presented on Russian in publications of the author [17] and in [5] and, briefly, on English in [3]. In addition, an English-language publication is currently being prepared with a detailed description of these very important and informative experiments.

The reasons that delay the timely publication of the results of this very important and effective experiment in physics journals are set out below in the Discussion of Results section.

\subsection{The Discussion of the Results}

It is important to note that the experiments presented in the article had the main goal of obtaining an answer to the question: does the effect take place within the framework of this technology, and if so, then conduct a detailed study of it in subsequent experiments. In addition, an important point here was testing working capacity the setup in real experimental conditions. Namely, the circumstances described above and the goals associated with the conduct of this experiment are an explanation for the author's insufficient attention to such an im- 
portant parameter of a possible gravitational manifestation as its sign.

Changes in the weights of the cargos found in Experiment II, according to the author, indicate the presence of a matched eddy electromagnetic current in the superconducting winding of the coil, which is realized in the process of its untwist and manifests itself through the vortex electromagnetic (gravitational) field. The observed effects are related precisely to the dynamics of superconductors, since preliminary tests performed on the facility at temperatures of 293 and $80^{\circ} \mathrm{K}$ were negative.

Below in this chapter important aspects related to this study are considered, the clarification of the circumstances of which contributed to the successful interpretation of the experimental results by the author.

1) First of all, the manifestation of gravitational effect at the stage of untwist the coil was unexpected. It was assumed that this effect should be expected at the stage of braking. This "strangeness" can be explained by the differences in the conditions of confinement of magnetic and electric charges at the indicated stages of the experimental process. Before untwist the coil the conditions of confinement of magnetic and electric charges in the conduction band of the superconductor are presented to be quite close, what suggests the possibility of realizing a stable mutual ordering of charges in the eddy electromagnetic current during the movement the superconductor lattice. It is under such conditions that one can expect the formation of a stable EM-current and, consequently, the manifestation of the gravitational effects in the form of the change in the weights of the cargos. Under the conditions of the maximum revolutions of the coil and keeping it in this rotation, the conditions for holding magnetic and electric charges in the superconductor lattice can differ significantly, what will prevent the formation of a stable matched EM-current.

2) The value of the change in the weight $\Delta \mathrm{P}$ of the tungsten cargo, noted in the experiment, significantly exceeds the corresponding value noted for the lead cargo, which testifies in favor of the gravitational nature of the observed effects (specific weights: $\mathrm{W}-19.30, \mathrm{~Pb}-11.34 \mathrm{~g} / \mathrm{cm}^{3}$ ). At the same time, it was noted that for equal conditions for the manifestation of the gravitational effect, the change in the weight of the lead weight would have to be $70.5 \mathrm{mg}$, if we take into account that for tungsten this change is $120 \mathrm{mg}$. By the explanation of the latter circumstance is the exit of the upper section of the coil from the superconducting state. The weights of the coil winding sections, measured after the experiment, were: $10.2 \mathrm{~kg}$ for the lower section and $5.1 \mathrm{~kg}$ for the upper section. Thus, the version of disabling the upper section from the realization of the effect is quite possible, since the calculated gravitational effect with its participation could be $47 \mathrm{mg}$. Recall that the value of the measured $\Delta \mathrm{P}$ for the lead cargo in the third cycle of Experiment II was $50 \pm 15 \mathrm{mg}$.

3) The collision noted in Chapter 2.2 with of the manifestation of the so-called the "strange tendency" in the 4th cycle of Experiment II requires a thorough explanation. As noted above, Experiment II, which followed negative Experiment I, had its main purpose to determine whether there was any effect within 
the framework of this experiment or not? Therefore, all attention during visual observation of the scale weights was focused on fixing the magnitude of the change in the weight of the cargos $(\Delta \mathrm{P})$ and of the time parameters of the process of these changes. At the same time, the sign of the change in the weights $\Delta \mathrm{P}$ of the weights remained outside the author's line of attention, under the conditions of a rapidly running experiment, i.e. the question of what takes place in this experiment: the attraction of cargo by a coil or their repulsion. The last question did not seem to the author to be any significant, considering that this experiment was carried out in 1981. At that time, the author was firmly convinced that the only possible gravitational manifestation here could be solely the attraction of the cargo to coil, in accordance with the law of the world gravitation. In addition, a feature of the scale of the balance used was that zero division of the scale and values $\Delta \mathrm{P}$ of the weight changes of the cargos in cycles 1 and 3 , did not fall into the single field of view of the observer. As for cycle 4 in Experiment II, so the magnitude of the change in the weight of the lead cargos $\Delta \mathrm{P}$ and the zero division of the scale of the balance were within the framework of a single visual field, what and led the author to discover of the noted "anomalous tendency". The subsequent resolution of the issue with the "anomalous tendency", which lasted for more than 20 years, led the author to the discovery of such important states of the gravitational field as paragravitation and ferrogavitation, as well as to effect of the gravitational (ferrogravitational) levitation [18] [19].

4) Due to the large mass of substance participating in the presented dynamic experiment, as well as the relatively small $\Delta \mathrm{T}^{\circ} \mathrm{K}$ between the temperature of liquid helium $\left(4.2^{\circ} \mathrm{K}\right)$ and the temperature of the superconducting transition of lead $\left(7.2^{\circ} \mathrm{K}\right)$, gravitational effects which manifested in the Experiments, according to the author are turned out to be in an acute dependence on local temperature conditions in the volume of lead windings. Very critical condition for the realization of the expected electromagnetic current is the provision of superconducting conditions at the points where the ends of the coil windings were closed (fused). It is the local exit of parts of the coil winding from the superconductivity mode that, according to the author, is a possible reason for the absence of the effect in Experiment I, as well as in cycles 2 and 5 of Experiment II. Local violations of the superconducting state regimes in the coil windings can also serve as an explanation for the observed mismatches in the measured $\Delta \mathrm{P}$ values in the effective cycles of Experiment II.

Note 3. It should, with regret, add that the author was unable to continue his experiments with rotating superconductors within the framework of the experimental technology described above and, therefore, to repeat and investigate the discovered effects, in result of change his working situation in 1982. This change was, in many ways, a consequence of his so-called "vicious hobby" for the search for real magnetic charges in atoms and substance. Under new circumstances the author was able to continue his research, but in a more modest experimental scales and was able, using other approaches, to explain the real physics of the "anomalous tendency" discovered in Experiment II. It should be added that it is 
precisely the life circumstances noted above that are the reason that these subsequent studies by author stretched out for more than 20 years.

The introduction of real magnetic charges, as well as true anti-electrons into basic physics allowed the author to formulate the concept of the World Physical Triad (WPT) which is extremely important for physical science, according to which the real World formed by three fundamental phases: Matter, Antimatter and Energo-phase (Energo-medium). This concept introduces into Physics the such a physical essence as "Dark energy", i.e. a global force factor responsible for all effects on particles and masses existing in Nature. The WPT concept, as well as the important implications for fundamental physical theory arising from it, is briefly presented in Chapter 5 .

\section{Other Experiments with Rotating Superconductors}

It is important to note that later and independently of the results of the experiments of the present author presented in the article experiments with rotating superconductors were performed by: Podkletnov E. and Nieminen R. in 1992 [20], as well as Tajmar M. and de Matos C. in 2003 [21]. In these experiments, according to their authors, gravitational effects were obtained.

In [20] the experimental gravitational field was obtained using a rotating superconducting metal-ceramic disk in strong magnetic field. Our analysis of the results of these experiments suggests that the effects observed are of gravitational nature. Under the conditions of these experiments, an eddy electromagnetic current is formed in the superconductor, i.e. a system of polarized S-gravitons that manifest themselves through an experimental gravitational field. It is important to note that the gravitational field generated in experiments [20] is the ferrogravitational field, which manifests itself in the processes of repulsion of adjacent masses from the superconducting disk. In essence, this effect is the effect of gravitational levitation, discovered by the present author and described in his publications and in this article (see Chapters 4 and 5).

The gravitational manifestations in the experiment of Tajmar M. and de Matos C. [21], which were detected during the accelerated rotation of the superconducting niobium ring, were interpreted by the authors as a manifestation of the gravimagnetic field. According to the interpretation of the author of this article, noted in [21] so-called gravimagnetic effects are manifestations of the real gravitational, or more precisely, the ferrogravitational field. The latest results are in good agreement with the gravitational effects obtained in the studies of the present author. It should also be noted that the magnitudes of gravitational effects obtained in the author's experiments are more than $10^{3}$ times higher than the effects found in the study of Tajmar M. and de Matos C.

Experimental and theoretical studies of the author of the article related to the discovery and detailed study of real magnetic charges existing in atoms and substance have shown that the gravitational field is a vortex electromagnetic field and is formed in joint vortex processes of electric and magnetic charges (see Chapter 4). 
However, the authors of the above other experiments when interpreting the results obtained, did not involve magnetic poles in their explanations, i.e. they, de facto, remained within Maxwell's flawed electromagnetic concept.

Note 4. In this article, the author named the date of his experiments with rotating superconductors 1979-1982. At the same time, the first official publication of their results was made only in 2001 (see [2]). The above date of these experiments, if any questions arise, can be confirmed by the technical specialists who participated with the author in the conduct of these experiments. The author presented the personalities of these specialists in the Acknowledgments section of this article.

\section{Paragravitation and Ferrogravitation, Ferrogravitational Levitation, Physical Nature of Mass}

The results of the experiments presented in the article, as well as other experimental and theoretical studies of the author, allowed him to establish the physical parameters and the place of real magnetic charges in the structures of atoms and matter. For example, the author showed that atomic shells consist of electric and magnetic spinor particles, the charges of which meet the condition $\mathrm{e}=\mathrm{g}$, and the shells themselves are electromagnetic, not purely electronic, as has always been believed [22] [23]. It is also important to emphasize that the number of magnetic spinor particles in atomic structures is approximately equal to the number of electrical particles. It is the electromagnetic shells of atoms that are natural sources (generators) of the gravitational field, which, in reality, is a vortex electromagnetic field. The elementary source of the gravitational field in the composition of atomic shells is a spinor electromagnetic quasiparticle, which received the author's name S-Graviton (S from Engl. a source). The S-graviton contains two spinors (an electron and a magneton) and two corresponding antispinors. The S-graviton can be represented as as a copula of magnetic and electric dipole revolving in antiphase in the same atomic orbit. This quasiparticle can also be represented in the form of two joint antiphase orbital currents of electric and magnetic charges.

The above-described model of an elementary vortex EM-current or S-Graviton can be written in mathematical form as: $\operatorname{rot}\left[J_{\mathrm{e}}-J_{\mathrm{g}}\right]$, где $J_{\mathrm{e}}$ и $J_{\mathrm{g}}$ are the vectors of the instantaneous current density of electric (e) and magnetic (g) charges corresponding to their vortex (circular) flows. Then the equation of the process of formation of the gravitational field is written in the following form:

$$
k \operatorname{rot}\left[\boldsymbol{J}_{\mathrm{e}}-\boldsymbol{J}_{\mathrm{g}}\right]=\operatorname{rot}[\boldsymbol{E}-\boldsymbol{H}],
$$

where $E$ and $H$ are the vectors of instantaneous electric and magnetic field strengths at each point of the vortex electromagnetic (gravitational) field, and $\mathrm{k}$ is the proportionality coefficient. The vortex vector $\operatorname{rot}[E-H]$ corresponds to an elementary quantum of the gravitational field emitted by one S-Graviton.

The minus signs, set in the above equation of the process of formation of the 
gravitational field (4), correspond both to the antiphase relation between the eddy currents of electric and magnetic charges in the S-Graviton, and to the antiparallel orientation of vectors of instantaneous strengths electric and magnetic fields at each point of the gravitational field. It is the minus signs, set in the above Equation (4), that correspond to the minimization of "Dark energy", which is realized in the Energy-phase under the influence of the fields of the S-graviton, presented in the article [24]. Thus, in contrast to the vortex magnetic field, each point of which corresponds to one vector of instantaneous intensity $H$, each point of the elementary gravitational field corresponds to two vectors of instantaneous field strengths $E$ и $H$, equal in magnitude and directed antiparallel to each other.

Vector-vortex analogy between magnetic (a) and gravitational (b) of fields is shows in Figure 4. Unlike from vortex magnetic field of every point of which is answered one vector of instantaneous tension of $H$, of every point of the elementary gravitational field is responsible two vectors of instantaneous tension of the fields of $E$ and $H$ equal by value and oriented antiparallel to each other.

If polarization of vortex vectors $\operatorname{rot}\left[J_{\mathrm{e}}-J_{\mathrm{g}}\right]$ of S-gravitons is realized in the structures of physical masses (atoms, nucleons, substance, etc.), what is accompanied by polarization of vortex vectors $\operatorname{rot}[E-H]$, then in analogy with magnetic fields ferromagnetics the gravitational fields being emitted by these masses can be called a ferrogravitational fields (FGF). The gravitational field formed by physical masses in the absence polarization of the vortex vectors of s-gravitons in their structures is a tensor or quasi-scalar field. And again, in analogy with magnetism, such field can to define as paragravitational field (PGF). Vector

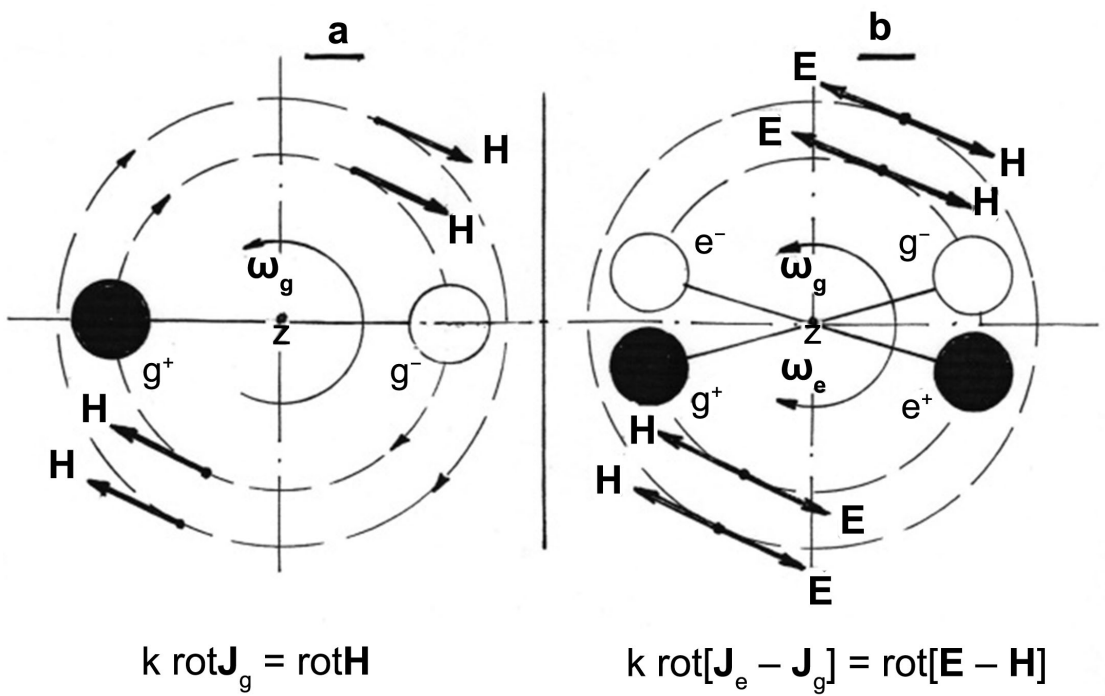

Figure 4. Schemes of mechanisms formation of the vortex spinor fields: magnetic (a) and electromagnetic (gravitational) (b). By white circles in the figure showed negative charged electric and magnetic spinors: electron $\left(\mathrm{e}^{-}\right)$and magneton $\left(\mathrm{g}^{-}\right)$and black circles-positively charged antispinors corresponding to them with charges $\mathrm{e}^{+}$and $\mathrm{g}^{+}$. The classical equations of the processes formation magnetic and electromagnetic (gravitational) of fields also show in the figure. 
conditions corresponding to these fields are written in the following form: $<\operatorname{rot}[E-H]>\neq 0$ for ferrogravitational field and $\langle\operatorname{rot}[E-H]>=0$ for the field paragravitational.

Since 2001 (see [2]), the author in his publications tried to explain that all varieties of Physical Mass (PM) are electromagnetic structures atomic type, consisting of electric and magnetic spinor particles. One of the main characteristic properties of all varieties of PM is their ability to emit a gravitational field that is formed as the result of joint orbital currents of electric and magnetic charges and is the vortex electromagnetic field. Thus, all types of physical mass (atoms, nucleons, substance etc.) are dynamic electromagnetic structures that emit a gravitational field and consist of electric and magnetic spinor particles. In other words: everything that creates a gravitational field is Mass. If magnetic poles (magnetic charges) are removed from the FM composition, then only electric particles will remain in this "place" which themselves are not able to form either mass or gravitational field.

At the same time, in modern mathematical physics, in conditions of complete disregard for real magnetic particles, the function of endow particles Mass entrusted to Higgs field, the quantum of which is his known boson [25]. The paradox of the situation with such "endow" is that the existing representation of mass is purely mathematical. Any real, general physical representation of the essence of FM is absent in modern physics. Therefore, the process of imparting mass to spinor particles is also mathematical and, as shown by the research of present author, has nothing to do with reality.

It is important to emphasize that Physical Mass and, for example, Matter are completely different of physical categories. The physical masses are, for example, nucleons and atoms. It is important to note that the individual spinor particles, as electric so and magnetic, are massless, because the mass is the result of their joint "structural operations". For example, the electron is massless particle and no "divine bosons" can't give him Mass. It should also be added that substance, atoms, nucleons i.e. the physical masses cannot be identified with Matter, as is now accepted in modern physical science.

The physical masses, emitting ferrogravitational field is push off from sources paragravitational field, for example, from the Earth. The last action is a manifestation levitation effect, which was first discovered by the author of this article and very widely distributed in Nature. In principle, such effects as gravitational volatility of atoms (molecules) of light gases, for example, hydrogen and also convectional processes in liquids and gas media should be also attributed to the natural levitation manifestations. The fact of participation of the gravitational levitation in the volatility of atoms (molecules) of hydrogen was detected in the experiment performed by the author of this article (see [19]). The levitation forces, which is caused by natural ferrogravitational fields, emitting by cells, are responsible for vertical growth (development) of plants and trees, as well as maintain them in an upright position. Namely, the forces of gravitational levita- 
tion are responsible for the amazing "aerobatics" of bumblebees, and also allow swifts and migratory birds to stay in the air for a long time.

Results of experiments author with magnetic charges and their currents in superconductors has allow him to develop technology to produce of the technical ferrogravitational field (FGF). Technical FGF and levitation forces formed by them can be used in transport, lifting and space engineering, in power engineering, as well as in numerous Gravito-Physical and Gravito-Chemical technologies (see publications author's [26] [27] [28]).

\section{World Physical Triad and "Dark Energy" in the Physics of Gravitational Influences}

The detection and multi-year research of the real magnetic charges in atoms and substance allowed the author to formulate the conception of the World Physical Triad (WPT), according to which the Real World consists of three fundamental phases: Matter, Antimatter and Energo-phase (Energo-medium) [29] (см. также [23]). All spinor particles with negative charge belong to Matter, and particles with a positive charge constitute Antimatterial phase. Such names of spinor particles as "lepton" and "antilepton", as well as "fermion" and "antifermion" are determined by the natural essence of the particles that is their material or antimaterial nature.

Note 5. It should be noted that in existing physical concepts, for example, atoms or nucleons are often referred to as matter. In his articles the author tries to explain that atoms, nucleons are not is matter at all. These structural formations consisting of material and antimaterial particles with electric and magnetic charges are, exclusively, of Physical masses. In addition, such physical essence as Antimatterial phase under no circumstances can it be can't be antisubstance.

The Energo-phase (Energo-medium) is superhigh-density of gas-like medium that fills the entire World Space and consists of its own massless and spinless particles, which are called the Energions (author's title) and are designated as $\varepsilon$. It is important to note that the Energo-phase can also be referred to as Energo-ether can find the points of contact between the Energo-phase and the Physical vacuum.

The energetic derivative from the Energo-phase is the so-called "Dark energy" ("DE") which is determined by the non-equilibrium states of Energo-phase in the form of local pressure fields $\left(\mathrm{P}_{\varepsilon}\right)$ created by the Energions.

Figure 5 shows the scheme of the World Physical Triad which includes three fundamental world phases: Matter, Antimatter and Energo-medium (Energo-phase). The fundamental particles that constitute the Phases of the Triad are indicated on the diagram, i.e. the spinors, antispinors and energions. In Figure 5 marked and such derivatives from the phases of Triad, as the spinor fields and "Dark energy". Outside of this scheme remain are condensates consisting of spinor particles of Matter and Antimatter, called Masses. 


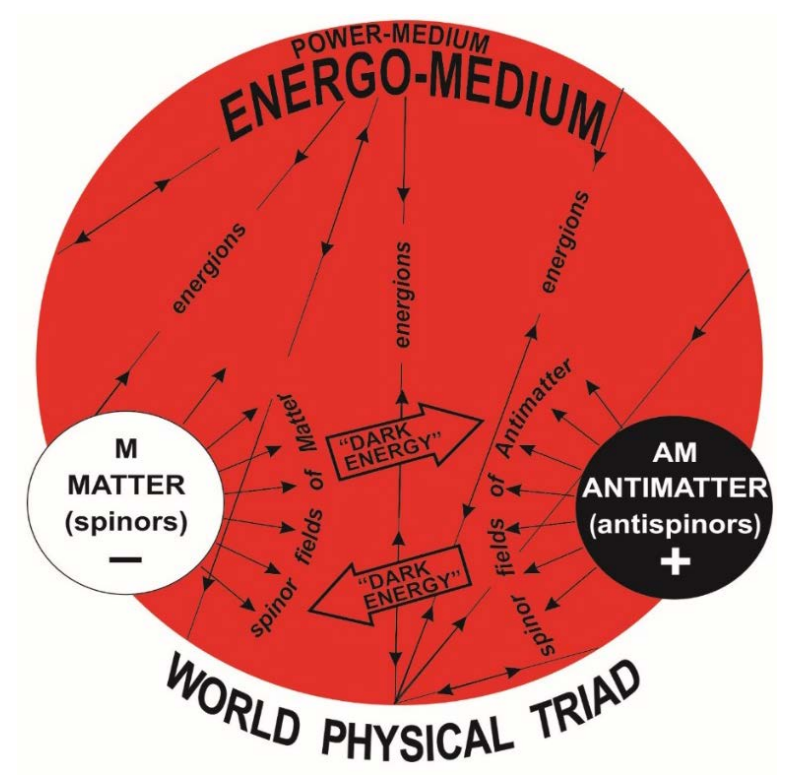

Figure 5. The diagram of the world physical triad.

The formations of "Dark energy" induce by spinor fields, i.e. fields of charged particles. Under this all varieties of spinor fields, including gravitational fields, themselves have no have essential of the force value. They are only intermediaries that influence on the local energy state of Energo-medium and induce the formation of "Dark energy" in it. Namely the "Dark energy" is global force factor which performs all the power acts with particles and masses, both in the scales of cosmos (the motion of galaxies, stars, planets, etc.), so and in the microcosm (the dynamics and so called interactions between Atoms and Charged Particles in compositions of Mass).

Between the Masses (atoms, bodies and other) which emit paragravitational field implemented the so-called the attraction or gravity. It is this last process, which is carried out, most often, by the forces of the gravitational "Dark energy" is the determining process in the physics of chemical bonding.

The Masses which emit the ferrogravtational field are repulsed from massessources of the paragravitational field, for example, from Earth, what constitute effect of the Gravitational levitation (GL) discovered and researched by author present article. An example of the so-called the ferrogravitational atom, i.e. atom emitting the FGF, is protium which is also called light hydrogen (designated as $\left.{ }^{1} \mathrm{H}\right)$.

The measure of the gravitational mass is the magnitude of the force of the gravitational "Dark energy" ( $G$ "DE") which is realized, for example, in pairs of bodies or atoms. The equation of the so-called Law of universal gravitation should be written in the form $F_{D E}= \pm G \frac{M_{1} \cdot M_{2}}{r^{2}}$, where the + sign refers to the case when the gravitational "DE" is implemented in of the pairs atoms or bodies emitting PGF. The masses, in this case, are pressed to each other by the force of the gravitational "DE" what is mistakenly perceived as their attraction. If the ef- 
fect of gravitational levitation is realized between bodies or atoms what, as a rule, takes place in a pair of masses emitting PGF and FGF, then before the constant $G$, in the above equation, a minus sign is set. In reality, of course, the gravitational constants, in the two cases of gravitational interactions noted above, will differ. Therefore, different symbols should be used for their designation. So, for example, the letter L (from the word "levitation") can be used to denote the constant of interactions between paragravitational and ferrogravitational masses which refers this new concept to processes of gravitational levitation.

It is important to note that the forces of the gravitational "DE" between masses over very small distances (e.g. less than 1 Angstrom) are not sufficiently studied. There is high probability that on small distances between the sources of the gravitational fields, the forces of the gravitational "Dark energy" are comparable with forces so called of electrostatic attraction and can even exceed its. In connection with the physical essence of "Dark energy" presented in the article and its global participation in the force effects on particles and masses, the term "interaction" between particles and masses does not correspond to the real state of affairs and is exclusively fictitious.

The reason that were closed all the possibilities of approaching of physical science to explaining real processes associated with the action of the gravitational "Dark energy" is the perverse performance about actual physics of the gravitational field.

As is generally known, in the existent official physical approaches, the gravitational field, more than one hundred years, binds with curvature of the four-dimensional space-time. In addition, a limiting factor here was the lack in of the representations of World Physical Triad which, according to the author, to a greater extent than the Physical vacuum or traditional Ether is meets the Reality.

\section{Conclusions}

Magnetic spinor particles (magnetic charges) that actually exist in the structures of atoms and substance are not recognized in the official physical theory and are not included in the standard model. The results of the author's research have shown that the basic reasons responsible for ignoring the noted fundamental particles by the world physical theory, for almost 150 years, are the special conditions of confinement of these particles in substance, which is radically different from the confinement electrons. In addition, the vicious concept of electric magnetism Maxwell, as well as such a "magnetic" surrogate as the theoretic monopole of Dirac, played a very negative role here. It is exclusively under the influence of these vicious concepts that the world's physical theory has ignored the existence in atoms and substance, practically, the three quarters of fundamental spinor particles, have left yourself only negative electric and neutral particles for "convenience" of physical interpretations

The investigations of F. Ehrenhaft and the author of the article allow us to assert that ignoring in physics real magnetic spinor particles, as well as true an- 
ti-electrons in the structures of substance and following the vicious teaching of Maxwell, is a tragic delusion of the existing official physical theory. Namely a mysticism that Maxwell laid down in the process of forming magnetic field, is responsible for the occurrence of such global theoretical misconceptions as the curvature of 4-dimensional space-time, which explains the phenomenon of gravity; the global expansion of the Universe and the Big Bang; the Annihilation of particles in particle-antiparticle pairs; the introduction of a fictitious Higgs boson to "endow" particles with mass and a great many others.

The vicious electrical magnetism of Maxwell, given its general-physical harmfulness, can associate with the "cancerous formation" in the "body" of physical science, the "metastases" of which have hit its many areas and directions. Conducting global "chemotherapy" of modern physical theory with the purpose of eliminating the numerous misconceptions generated by Maxwell's vicious concept, according to the author, is the most urgent task for today. All this needs to be done as soon as possible, because another 10 - 15 years and even robots will laugh at us.

\section{Acknowledgements}

The author expresses deep gratitude to Elena Sizova and Vitaly Sizov who for many years helped and supported him which largely contributed to the implementation of his research activities.

The author is grateful to the technical specialists: Alexander Davydov, Nikolai Koshelev, Alexander Lomakin, Evgeni Moiseenkov and Sergei Staritsyn who together with him conducted the main experiments connected with the discovery and investigation of real magnetic charges.

\section{Conflicts of Interest}

The author declares no conflicts of interest regarding the publication of this paper.

\section{References}

[1] Sizov, R.A. (1971) Journal of Experimental and Theoretical Physics, 60, 1363-1370.

[2] Sizov, R.A. (2001) New Presentation of Nature Magnetism, Gravitation and Nuclear Forces of Bonding. Akademizdat Center "Science", Moscow, 62 p.

[3] Sizov, R.A. (2015) Journal of Modern Physics, 6, 1013-1022. https://doi.org/10.4236/jmp.2015.68106

[4] Sizov, R.A. (2020) Journal of Modern Physics, 11, 1245-1261. https://doi.org/10.4236/jmp.2020.118078

[5] Sizov, R.A. (2008) Electric and Magnetic Spinor Particles as Structure Forming Components of Mass and Electromagnetic Source Gravitation. Akademizdat-Center "Science", Moscow, 260 p.

[6] Sizov, R.A. (2020) OSP Journal of Nuclear Science, 2, JNS-2-111.

[7] Maxwell, J.C. (1873) Treatise on Electricity and Magnetism. Volume 1-2, Clarendon Press, Oxford. 
[8] Sizov, R.A. (2019) Applied Physics Research, 11, 49-55. https://doi.org/10.5539/apr.v11n3p49

[9] Sizov, R.A. (2019) Applied Science and Innovative Research, 3, 257-263. https://doi.org/10.22158/asir.v3n4p257

[10] Ehrenhaft, F. (1910) Wiener Berichte 119(11a) 836; (1942) Jour. Frank. Inst. Mar., 235; (1951) Acta Phys. Austriaca, 5, 12.

[11] Mikhailov, V.F. and Ruzicka, J. (1989) Acta Physica Universitatis Comenianae, 29, 97-148.

[12] Sizov, R.A. (2018) Journal of Modern Physics, 9, 145-171. https://doi.org/10.4236/jmp.2018.92010

[13] Dirac, P.A.M. (1931) Proceedings of the Royal Society A, 133, 60-72. https://doi.org/10.1098/rspa.1931.0130

[14] Sizov, R.A. (2015) Journal of Modern Physics, 6, 2280-2289. https://doi.org/10.4236/jmp.2015.615232

[15] Sizov, R.A. (2018) Physics \& Astronomy International Journal, 2, 17-20.

[16] Sizov, R.A. (2017) Journal of Modern Physics, 8, 964-981. https://doi.org/10.4236/jmp.2017.86061

[17] Sizov, R.A. (2007) Magnetic Fundamental Particles (Magnetons) in Physics of Magnetic and Electromagnetic Effects. Akademizdat-Center "Science", Moscow, 149 p.

[18] Sizov, R.A. (2012) Levitation as a Spread Force Manifestation of Natural Ferrogravitation. Akademizdat-Center "Science", Moscow, 96 p.

[19] Sizov, R.A. (2015) Journal of Modern Physics, 6, 1591-1601. https://doi.org/10.4236/jmp.2015.611161

[20] Podkletnov, E. and Nieminen, R. (1992) Physica C. Superconductivity, 203, 441-444. https://doi.org/10.1016/0921-4534(92)90055-H

[21] Tajmar, M. and de Matos, C. (2003) Physica C: Superconductivity, 385, 551-554. https://doi.org/10.1016/S0921-4534(02)02305-5

[22] Sizov, R.A. (2016) Journal of Modern Physics, 7, 2374-2397. https://doi.org/10.4236/jmp.2016.716206

[23] Sizov, R.A. (2017) Journal of Modern Physics, 8, 1072-1086. https://doi.org/10.4236/jmp.2017.87069

[24] Sizov, R.A. (2016) Journal of Modern Physics, 7, 558-572. https://doi.org/10.4236/jmp.2016.76059

[25] Higgs, P.W. (1964) Physics Letters, 12, 132-133. https://doi.org/10.1016/0031-9163(64)91136-9

[26] Sizov, R.A. (2013) Transference of People and Loads in the Terrestrial Space by Means of Technical Ferrogravitation. Akademizdat-Center "Science", Moscow, 118 p.

[27] Sizov, R.A. (2020) Applied Science and Innovative Research, 4, 1. https://doi.org/10.22158/asir.v4n1p1

[28] Sizov, R.A. (2020) Open Journal of Physical Chemistry, 10, 48-67. https://doi.org/10.4236/ojpc.2020.101003

[29] Sizov, R.A. (2011/2012) Matter, Antimatter and Energo-Medium Is Physical Triad of the Real World. Akademizdat-Center "Science", Moscow, 192 p. 